Research Article

\title{
Knockdown of DIAPH3 Inhibits the Proliferation of Cervical Cancer Cells through Inactivating mTOR Signaling Pathway
}

\author{
Linling Wan $\mathbb{D}^{1},{ }^{1}$ Jiamin $\mathrm{Zhu}^{2}$ and Qunying $\mathrm{Wu} \mathbb{D}^{1}$ \\ ${ }^{1}$ Department of Obstetrics and Gynecology, The Affiliated Jiangyin Hospital of Southeast University Medical College, Wuxi, \\ Jiangsu 214000, China \\ ${ }^{2}$ Department of Oncology, The Affiliated Jiangyin Hospital of Southeast University Medical College, Wuxi, Jiangsu 214000, China
}

Correspondence should be addressed to Qunying Wu; jywqying@163.com

Received 6 September 2021; Accepted 20 September 2021; Published 6 October 2021

Academic Editor: Song Cao

Copyright (c) 2021 Linling Wan et al. This is an open access article distributed under the Creative Commons Attribution License, which permits unrestricted use, distribution, and reproduction in any medium, provided the original work is properly cited.

Cervical cancer (CC) ranks fourth for both incidence and mortality among females in worldwide. Therefore, it is urgent to explore new therapeutic and diagnostic targets for cervical cancer. Diaphanous-related formin 3 (DIAPH3) has been identified to play crucial roles in many malignant tumors. But its function and potential mechanism in CC remain largely unknown. In our study, DIAPH3 was frequently upregulated in CC tissue samples and increased expression of DIAPH3 was associated with poor overall survival according to several databases. Through in vitro and in vivo experiments, we found that decreased expression levels of DIAPH3 significantly inhibited the progression of CC. The GSEA analysis and western blot assay indicated that DIAPH3 was associated with the mTOR signaling pathway. The univariate and multivariate Cox analysis indicated that DIAPH3 was an independent prognosis risk factor in TCGA-CESC. And we confirmed that DIAPH3 expression was clearly related to tumor immune infiltrating cells (TIICs) by the analysis of CIBERSORT and TIMER databases. Taken together, we revealed that DIAPH3 plays as an oncogene through mTOR signaling pathway and DIAPH3 might be a potential prognostic biomarker in CC.

\section{Introduction}

Cervical cancer (CC) is a kind of common malignancies worldwide. And both the incidence and the mortality of CC remain fourth among females [1]. There is a substantial increase in cervical cancer incidence in China [2]. Most cases are often diagnosed at an advanced stage. Almost all cases are due to human papillomavirus (HPV) infection [3]. The treatments of CC include surgery or a concurrent chemoradiotherapy program composed of cisplatin-based chemotherapy with brachytherapy and external beam radiotherapy [4]. Despite the advancement of diagnosis and treatment technology, the five-year survival rates of advanced CC are about $30 \%-50 \%$ [5]. Hence, it is urgent to explore new therapeutic and diagnostic targets for cervical cancer.

The diaphanous-related formin 3 (DIAPH3) is considered a core modulator of the cytoskeleton [6] and the host gene locates on human chromosome 13q21.2 [7]. DIAPH3 is investigated to reshape the cytoskeleton [8] and regulates the expression of GSK3 $\beta$ to preserve microtubule stabilization [9]. Moreover, previous studies reported that DIAPH3 plays many crucial roles in many malignancies. Di Vizio D et al. confirmed that DIAPH3 facilitates the metastasis process of prostate cancer [10]. Jiang et al. verified that the overexpression of DIAPH3 suppresses the migration of triplenegative breast cancer [11]. Dong et al. reported that DIAPH3 facilitates the malignant biological behavior of HCC cells through activating $\beta$-catenin/TCF signaling pathway [12]. To our knowledge, the functions of DIAPH3 and its molecular mechanism in cervical cancer were largely unknown. In the present study, we revealed that DIAPH3 plays as an oncogene through mTOR signaling pathway. We revealed that DIAPH3 promoted proliferation through mTOR signaling pathway in cervical cancer.

In recent years, tumor immunity has gradually attracted the attention of many researchers. The regulatory $\mathrm{T}$ cells $(\mathrm{T}$ regs) could affect the role of cytotoxic $\mathrm{T}$ cells to facilitate 
tumor cells to get rid of the immune system [13]. A large number of papers have deeply explored the complex tumor immune regulation process and immune checkpoints to influence the process of antitumor immune response. For example, the advancement of antibody drugs against PD1 and PD-L1 for the therapy of prostate cancer and renal cell carcinoma has shown great efficacy in clinical practice [14-17]. Therefore, in this study, we further evaluated the prognostic value of DIAPH3 and assessed the association between the expression of DIAPH3 and tumor immune infiltration level in CC.

\section{Materials and Methods}

2.1. CC Tissues and Cells. The CC tissues were collected from the Department of Obstetrics and Gynecology, The Affiliated Jiangyin Hospital of Southeast University Medical College. All patients have signed informed consent. Human cervical cancer cell lines C33 A, Caski, Siha, and HeLa were purchased from the Cell Center of Shanghai Institutes for Biological Sciences. $\mathrm{HaCaT}$ cells were used as normal controls, which were obtained from Nanjing Kaiji Biotechnology Company. Cells were cultured in Dulbecco's modified Eagle's medium (Gibco, USA) supplemented with $10 \%$ fetal bovine serum (Gibco, USA) and $1 \%$ penicillin/streptomycin (Gibco, USA) at $37{ }^{\circ} \mathrm{C}$ with an atmosphere of $5 \% \mathrm{CO}_{2}$ in a humidified cell chamber.

2.2. $q R T-P C R$ and RNA Extraction. Firstly, we utilized the TRIzol reagent (Invitrogen, USA) to extract the RNA from $\mathrm{CC}$ cells. Then the reverse transcription process was carried out according to the instructions of PrimeScript RT Reagent (TaKaRa, Japan) and all PCRs were conducted with SYBR Premix Ex Taq Kit (TaKaRa, Japan) according to the manufacturer's instructions. $\beta$-Actin was used as a normal control. The $2^{\wedge \Delta \mathrm{CT}}$ method was used to quantify the relative expression. The involved primers were as follows: $\beta$-actin forward, 5"-GCATCGTCACCAACTGGGAC-3'; reverse, 5' -ACCTGGCCGTCAGGCAGCTC-3'; DIAPH3, forward, 5',-ACGGATGATATGCTGGACAA-3'; reverse, 5',-CAG TGGCTTTGGAAAGTTCT-3'. The experiment was performed in triplicate.

2.3. CCK8 Assay. We utilized the Cell Counting Kit-8 (Dojindo, Kumamoto, Japan) to assess the proliferation ability of CC cells. Cells in different treatment groups were seeded into 96-well plates (1,000 cells/well) and cultured with complete medium for 5 days. Then the cells were incubated with CCK-8 solution for $2 \mathrm{~h}$. Next, the proliferation ability was evaluated by OD value at $450 \mathrm{~nm}$. Each sample was performed in triplicate.

2.4. Colony Formation Assay. Cells in different treatment groups were seeded in 6-well plates (500 cells/well) and placed in the incubator for 2 weeks. After that, the cells were stained with crystal violet for 30 minutes. The numbers of colony formations were counted. The experiment was performed in triplicate.
2.5. EdU Assay. To further assess the proliferation ability, we also utilized the EdU kit (Beyotime Biotechnology, Shanghai, China). The treated cells were firstly plated into 96-well plates $\left(3 \times 10^{4} /\right.$ well $)$ and cultured for 24 hours. Then we added the EdU reagent $(50 \mu \mathrm{mol} / \mathrm{L})$. Next, cells were permeabilized with $0.5 \%$ TritonX-100 for 10 minutes after fixing in $4 \%$ formaldehyde for 2 hours. $1 \times$ Apollo reaction solution $(400 \mu \mathrm{L})$ reacted with EdU (Beyotime Biotechnology, Shanghai, China) for 30 minutes, and DAPI $(400 \mu \mathrm{L})$ stained the nucleus after washing with PBS for three times. Finally, the microscope was used to capture the images of cells. The experiment was performed in triplicate.

2.6. Western Blot Assay. The concentrations of the extracted protein were evaluated by BCA kit (Beyotime Biotechnology, Shanghai, China). After electrophoresis, the PVDF membranes were used. After blocking with 5\% nonfat milk at a shaker for 2 hours, we incubated the membranes with different specific primary antibodies at $4^{\circ} \mathrm{C}$ overnight. In another day, the membranes were incubated with the corresponding secondary antibodies for 2 hours. The antibodies were listed as follows: $\beta$-actin (CST, \#3700, 1:1000), DIAPH3 (Abcam, ab227276, 1:1000), AKT (Abcam, ab8805, 1:500), p-AKT (Abcam, ab38449, 1:1000), mTOR (Abcam, ab134903, 1:10000), PTEN (Abcam, ab32199, 1: 10000), and p-p70s6k (CST, \#9208, $1: 1000)$. All assays were performed in triplicate.

2.7. Animal Experiment. To establish the tumor growth models, we purchased four-week-old female $\mathrm{BALB} / \mathrm{c}$ nude mice. The transfected cells $\left(2 \times 10^{6}\right.$ cells $\left./ 100 \mu \mathrm{L}\right)$ with PBS suspension were subcutaneously injected. After 5 weeks, we sacrificed the mice and observed the tumor progression.

2.8. Analysis of Tumor Immune Infiltrated Cells (TIICs). We conducted the association analysis between the expression of DIAPH3 and the fractions of tumor infiltrated immune cells in CESC by using the data from the CIBERSORT and TIMER databases. The correlation was examined by Spearman test.

2.9. Statistical Analysis. The data were presented as mean \pm SD. We statistically analyzed the data through Student's $t$-test using GraphPad Prism 7. Survival analysis was calculated by Kaplan-Meier plots. $p$ values $<0.05$ represented a statistically significant difference. The association of DIAPH3 with clinicopathological characteristics was analyzed by the $\chi^{2}$ test.

\section{Results}

3.1. DIAPH3 Was Overexpressed in Cervical Cancer and Showed a Poor Prognosis. We first retrieved data of cervical cancer from the TCGA database, and we selected DAIPH3 as a potential oncogene in CC according to fold change $>2$ and $P$ value $<0.01$ (Figure 1(a)). To further confirm it in a large cohort of patient samples, we analyzed the expression of 


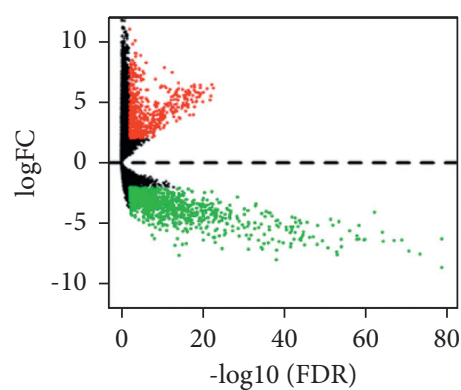

(a)

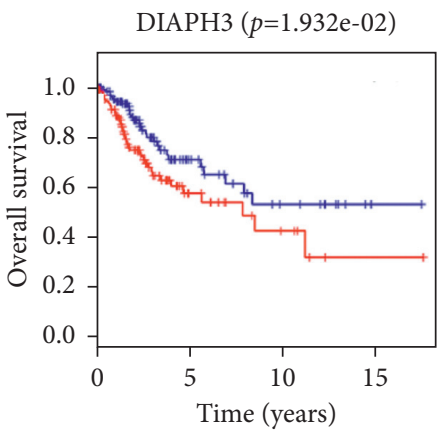

Low expression

_ High expression

(c)

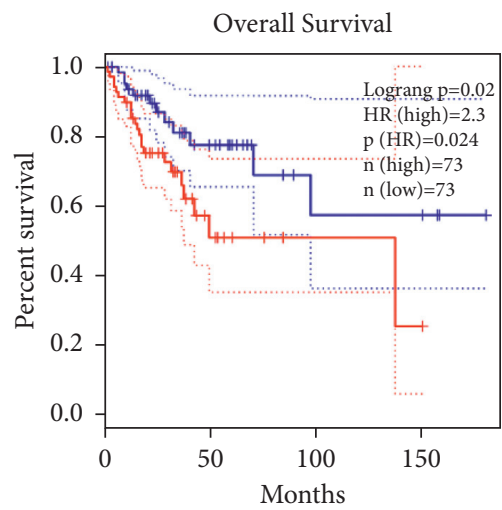

— Low DIAPH3 TPM

— High DIAPH3 TPM

(e)

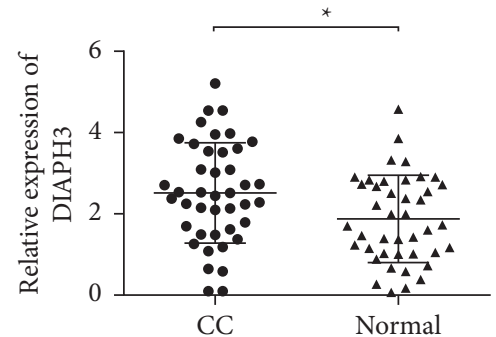

(g)

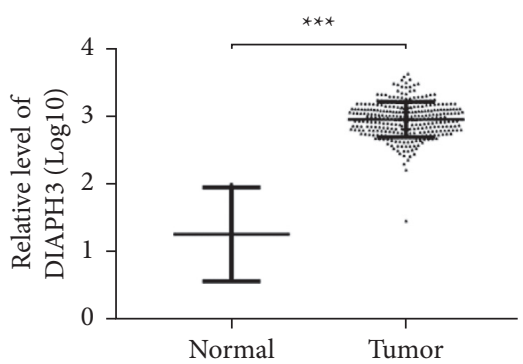

(b)

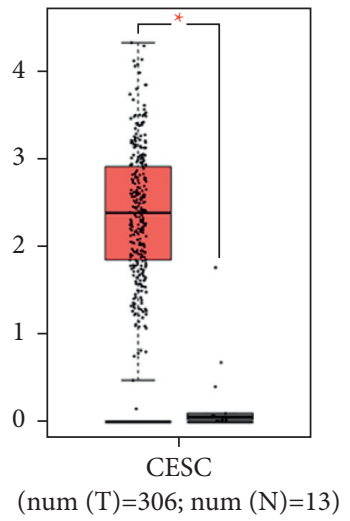

(d)

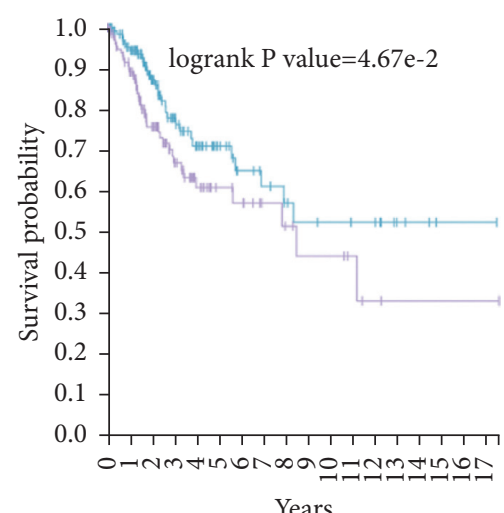

- Low $(\mathrm{n}=145)$

- High $(\mathrm{n}=146)$

(f)

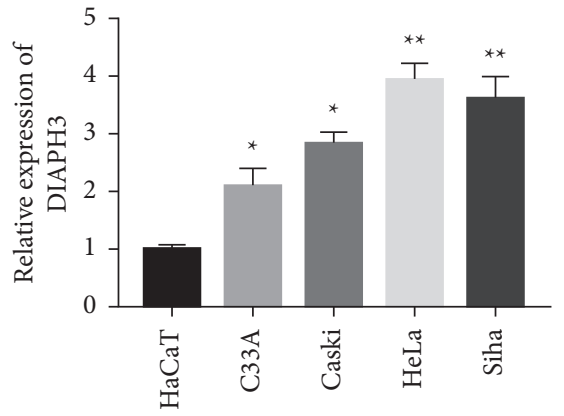

(h)

Figure 1: Continued. 


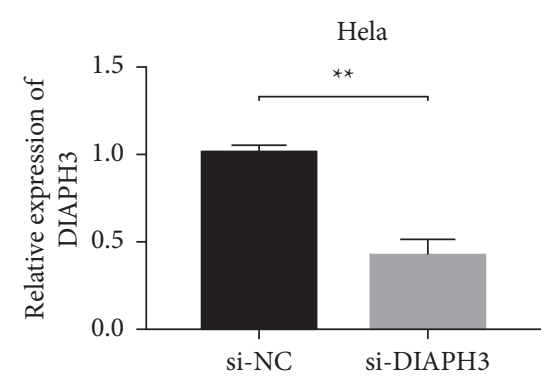

(i)

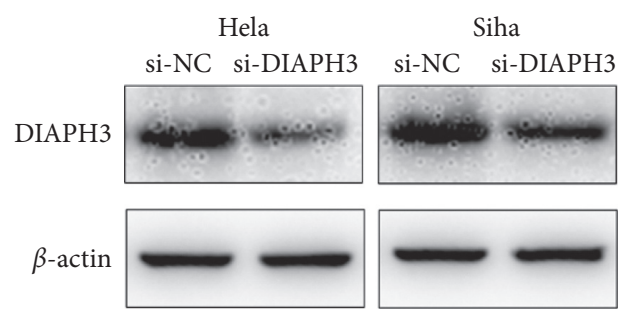

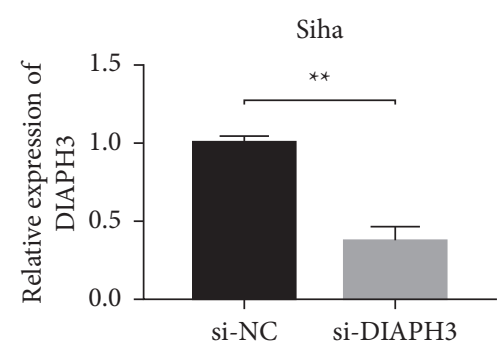

(j)

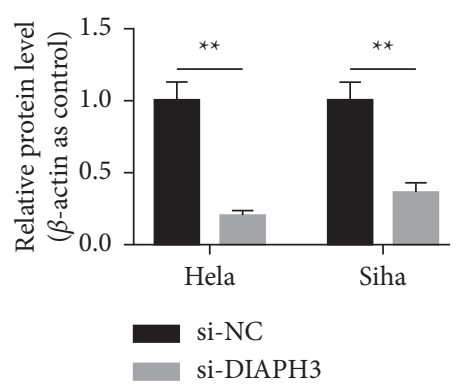

$(\mathrm{k})$

Figure 1: DIAPH3 was overexpressed in cervical cancer and showed a poor prognosis. (a) The volcano map of differential genes in cervical cancer in TCGA database. Fold change $>2, P$ value $<0.01$. (b) DIAPH3 was overexpressed in cervical cancer by analysis of TCGA data. (c) High expression of DIAPH3 displayed a poor overall survival in TCGA database. (d) The expression of DIAPH3 in cervical cancer according to GEPIA database. $(\mathrm{e}, \mathrm{f})$ The overall survival rates of DIAPH3 in GEPIA database and The Protein Atlas, respectively. (g) DIAPH3 was upregulated in CC cells compared with HaCaT. (h, i) The relative expression of DIAPH3 after transfection of si-NC and si-DIAPH3 in HeLa and Siha cells by qRT-PCR. ( $\mathrm{j}, \mathrm{k}$ ) The relative expression of DIAPH3 after transfection of si-NC and si-DIAPH3 in HeLa and Siha cells by western blot. $\beta$-Actin was used as an internal control.

DIAPH3 in CC in TCGA database, consisting of 305 tumor tissues and 3 normal tissues. It showed that DIAPH3 was significantly overexpressed in CC compared with normal samples (Figure 1(b)). Moreover, we found that high expression of DIAPH3 indicated a poor overall survival in CC according to TCGA database (Figure 1(c)). Simultaneously, we verified the above results through GEPIA database and it showed the similar outcome (Figures $1(\mathrm{~d})$ and $1(\mathrm{e})$ ). Then we added The Human Protein Atlas and we found that patients with high expression of DIAPH3 exhibited a lower overall survival rate (Figure 1(f)). Moreover, we performed the analysis of DIAPH3 expression with survival in CC by using the TCGA-CESC cohort. Combined with the univariate cox (Table 1) and multivariate cox analysis (Table 2), it indicated that the expression of DIAPH3 was an independent prognostic risk index of cervical cancer. And we found that DIAPH3 expression was significantly upregulated in 42 pairs of CC samples compared to normal cases (Figure $1(\mathrm{~g})$ ). To determine the functions of DIAPH3 in CC in vitro, we examined DIAPH3 expression in four CC cells (HeLa, Siha, C33 A, and Caski) and HaCaT cells (normal control). It was shown that DIAPH3 was increased in CC cells, especially in HeLa and Siha cells (Figure 1(h)), so we chose these two cell types for further investigation. Additionally, we transfected si-NC and si-DIAPH3 into HeLa and Siha cells, respectively, and we validated the knockdown efficiency of DIAPH3 using qRT-PCR and western blot assay. We observed that the expression of DIAPH3 was obviously decreased in the si-DIAPH3 group (Figures 1(i)$1(\mathrm{k}))$. Additionally, we evaluated the association between the expression of DIAPH3 and patients' pathological characteristics. In the group of tumor size larger than $3 \mathrm{~cm}$, the expression level of DIAPH3 was significantly upregulated (Table 3).

3.2. Knockdown of DIAPH3 Inhibited the Progression of CC In Vitro and In Vivo. To determine the biological functions of DIAPH3 in CC, we transfected si-NC and si-DIAPH3 into HeLa and Siha cells, respectively. After transfection, we utilized different means to detect cell proliferation in vitro. In CCK8 assay, it was shown that the ability of proliferation in si-DAIPH3 group was notably declined compared to that in si-NC group in HeLa and Siha cells (Figures 2(a) and 2(b)). In the colony formation assay, the results showed that knockdown of DIAPH3 weakened colony formation ability in HeLa cells (Figures 2(c) and 2(e)), and it showed similar results in Siha cells (Figures 2(d) and 2(f)). Then EdU incorporation assay was utilized for further assessment of the influence of DIAPH3 on CC cell proliferation. We found that the rate of EdU in the si-DIAPH3 group was obviously declined compared to that in si-NC group in HeLa cells (Figures 2(g) and 2(h)), and the tendency was consistent with that in Siha cells (Figures 2(i) and 2(j)). 
TABLE 1: Univariate Cox regression analysis of OS in TCGA-CESC cohort.

\begin{tabular}{lcccc}
\hline & HR & HR.95L & HR.95H & $p$ value \\
\hline Age & 1.012642719 & 0.967516855 & 1.059873294 & 0.589082707 \\
Grade & 1.347682976 & 0.60019885 & 3.026079445 & 0.469675039 \\
T & 1.571480239 & 0.791716299 & 3.1192362 & 0.196265045 \\
$\mathrm{~N}$ & 1.926434151 & 0.732327039 & 5.067610971 & 0.183955862 \\
DIAPH3 & 1.46513653 & 1.182831075 & 1.814819628 & $0.000469571^{* * *}$ \\
\hline
\end{tabular}

${ }^{* * *} p<0.001$ statistically significant difference.

TABLE 2: Multivariate Cox regression analysis of OS in TCGA-CESC cohort.

\begin{tabular}{lcccc}
\hline & HR & HR.95L & HR.95H & $p$ value \\
\hline Age & 0.997843949 & 0.952416863 & 1.045437753 & 0.927658286 \\
Grade & 1.045822636 & 0.461258359 & 2.371219872 & 0.914572354 \\
$\mathrm{~T}$ & 1.367948739 & 0.698662983 & 2.678378272 & 0.360744139 \\
$\mathrm{~N}$ & 2.418271676 & 0.852581947 & 6.859209164 & 0.096887932 \\
DIAPH3 & 1.518865321 & 1.184673829 & 1.947330823 & $0.000978608^{* * *}$ \\
\hline
\end{tabular}

${ }_{* * *} p<0.001$ statistically significant difference.

TABLE 3: Expression of DIAPH3 in cervical cancer according to patients' clinicopathological characteristics.

\begin{tabular}{|c|c|c|c|c|}
\hline \multirow{2}{*}{ Characteristics } & \multirow{2}{*}{ Number } & \multicolumn{2}{|c|}{ DIAPH3 expression } & \multirow{2}{*}{$P$ value } \\
\hline & & High-risk group & Low-risk group & \\
\hline \multicolumn{5}{|l|}{ Age (years) } \\
\hline$<50$ & 19 & 11 & 8 & \multirow{2}{*}{0.352} \\
\hline$\geq 50$ & 23 & 10 & 13 & \\
\hline \multicolumn{5}{|l|}{ Differentiation } \\
\hline Well/moderate & 26 & 12 & 14 & \multirow{2}{*}{0.525} \\
\hline Poor & 16 & 9 & 7 & \\
\hline \multicolumn{5}{|l|}{ Size $(\mathrm{cm})$} \\
\hline$<3$ & 24 & 8 & 16 & \multirow{2}{*}{$0.013^{*}$} \\
\hline$\geq 3$ & 18 & 13 & 5 & \\
\hline \multicolumn{5}{|l|}{ FIGO stage } \\
\hline I & 24 & 9 & 15 & \multirow{2}{*}{0.061} \\
\hline II and III & 18 & 12 & 6 & \\
\hline \multicolumn{5}{|c|}{ Lymph node metastasis } \\
\hline No & 25 & 13 & 12 & \multirow{2}{*}{0.753} \\
\hline Yes & 17 & 8 & 9 & \\
\hline
\end{tabular}

${ }^{*} p<0.05$ statistically significant difference.

To further investigate the functions of DIAPH3 on tumor development, we performed xenograft assays. The transfected cells were subcutaneously injected. After 5 weeks, we sacrificed the mice and obtained the tumor. The result indicated the tumor weight and volume in the siDiAPH3 group were obviously decreased in HeLa cells. And it exhibited similar results in Siha cells (Figures 3(d)-3(f)). Collectively, these results demonstrated that knockdown of DIAPH3 could suppress the progression of CC in vitro and in vivo.

\subsection{Knockdown of DIAPH3 Inhibited Cell Proliferation} through Suppression of mTOR Signaling Pathway. After that, we utilized bioinformatic analysis to determine the underlying mechanism of DIAPH3 on CC cell proliferation. We first analyzed bioprocess pathways by Gene Set Enrichment Analysis (GSEA). The results of Figures 4(a) and 4(b) demonstrated that high expression of DIAPH3 was positively correlated with the mTOR pathway $(P$ value $<0.01)$. Next, we used western blot assay for further investigation. In HeLa cells, it showed that downregulated level of DIAPH3 decreased the expression of related proteins $\mathrm{p}-\mathrm{AKT}$, mTOR, and p-p70s6k and increased the expression of PTEN in mTOR signaling pathway and there is a similar trend in Siha cells (Figure 4(c)).

Accordingly, these data indicated that knockdown of DIAPH3 inhibited the proliferation of cervical cancer cells through inactivating the mTOR signaling pathway.

3.4. Correlation between the Expression of DIAPH3 and the Fractions of TIICs in CC. To further probe the association between DIAPH3 expression and TIICs in CC, we analyzed the subgroups of tumor-infiltrating immune cells by analysis of the data from CIBERSORT. Then we constructed 22 kinds 


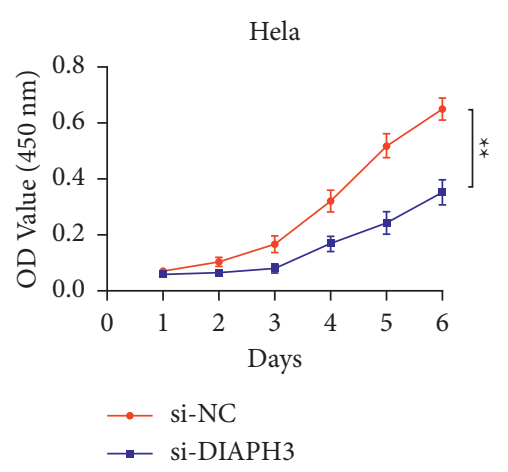

(a)

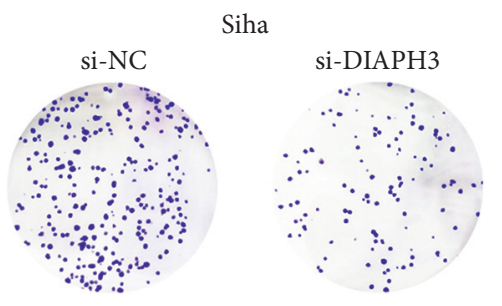

(d)

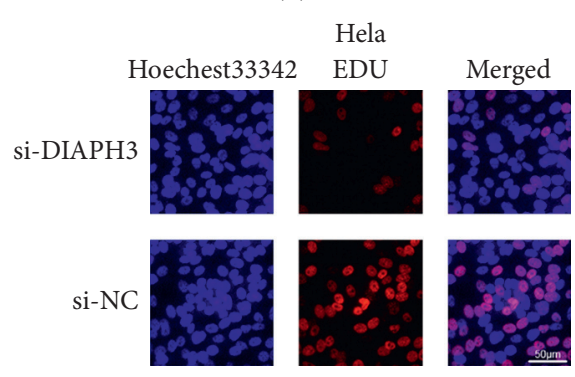

(g)

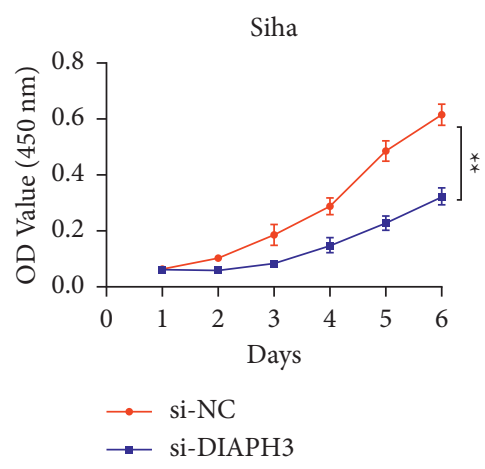

(b)

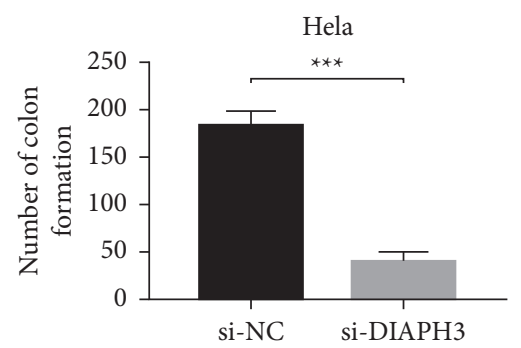

(e)

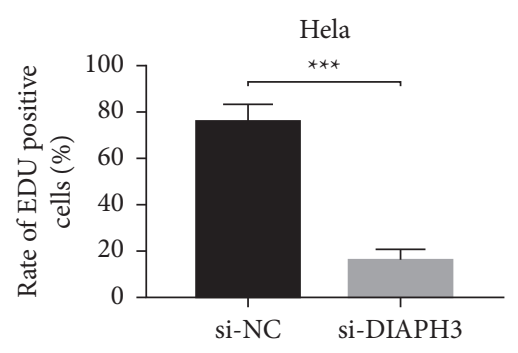

(h)

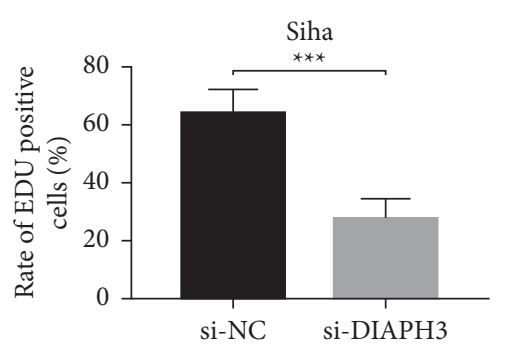

(j)

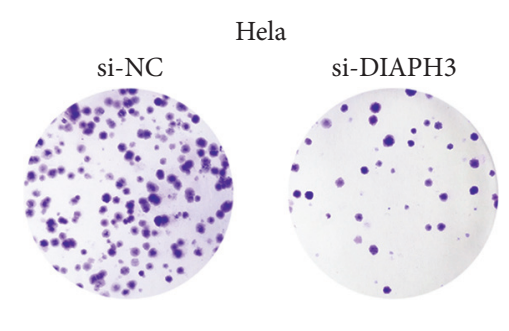

(c)

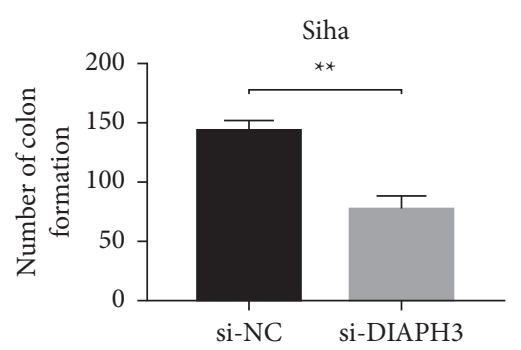

(f)

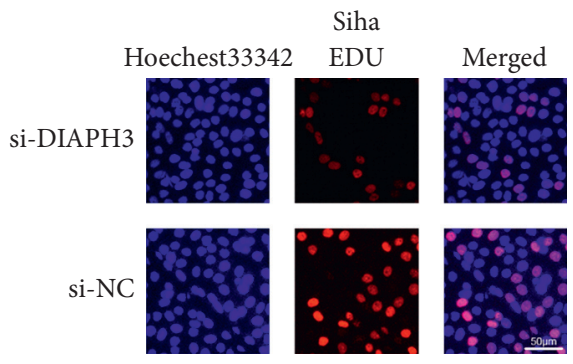

(i)

Figure 2: Knockdown of DIAPH3 inhibited cell proliferation in vitro. (a, b) Cell viability in si-DAIPH3 group was notably decreased compared to that in si-NC group in HeLa and Siha cells by CCK8 assay. (c, d) The representative images of colony formation assay after transfection in HeLa and Siha cells. (e, f) Knockdown of DIAPH3 decreased the number of colony formation in HeLa and Siha cells. (g, h) The rates of EdU-positive cells were declined in the si-DIAPH3 group compared with those in si-NC group in HeLa cells. (i, j) The rates of EdU-positive cells were declined in the si-DIAPH3 group compared with those in si-NC group in Siha cells.

of immune cell profiles (Figure 5(a)). As shown in Figure 5(b) and Table 4, the expression of DIAPH3 was positively associated with the abundance of acquired immunocytes ( $\mathrm{T}$ helper cells $(R=0.173, P=0.002)$, Th2 cells $(R=0.310, P<0.001)$, and NK cells $(R=0.115, P=0.045))$ and negatively related to the abundance of innate immunocytes (B cells $(R=-0.248, P<0.001)$, Treg $(R=-0.177$, $P=0.002)$, DCs $(R=-0.290, P<0.001)$, iDCs $(R=-0.243$,
$P<0.001)$, pDCs $(R=-0.162, P=0.004)$, cytotoxic cells $(R=-0.165, P=0.004)$, etc.). By differential correlation analysis, we observed that three types of TIICs were negatively associated with the expression of DIAPH3, including regulatory $\mathrm{T}$ cells (Tregs), naïve $\mathrm{B}$ cells, and Mast cells resting. But Mast cells activated were shown to be positively correlated with the expression of DIAPH3 (Figures 6(a) and 6(b)). 


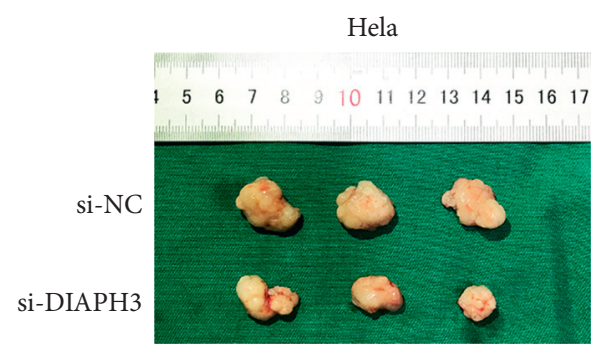

(a)

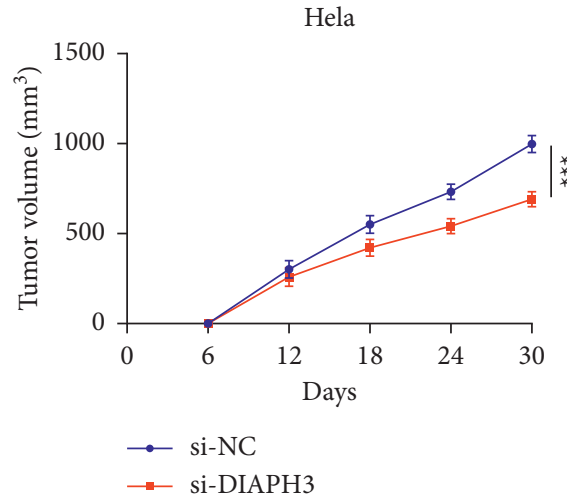

(c)

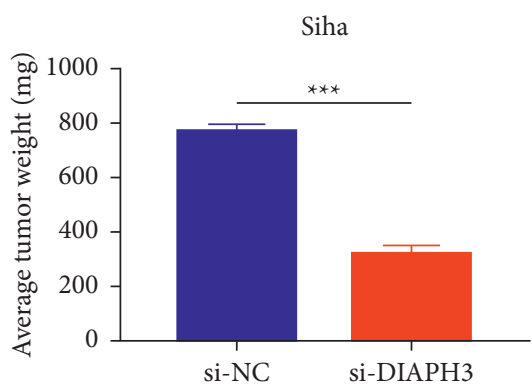

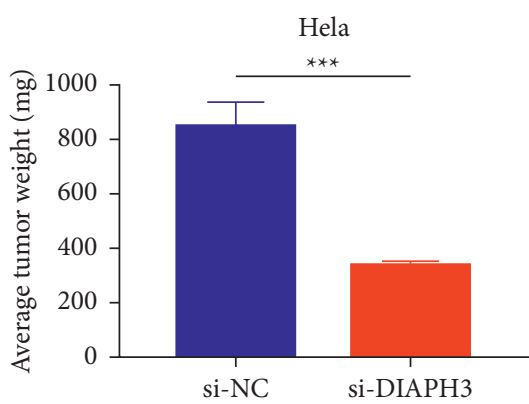

(b)

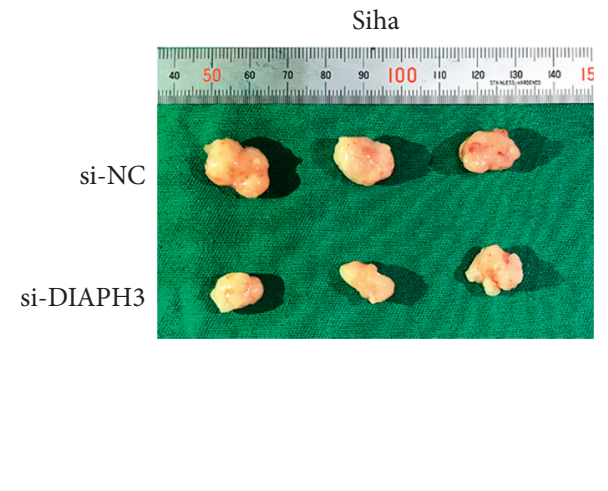

(d)

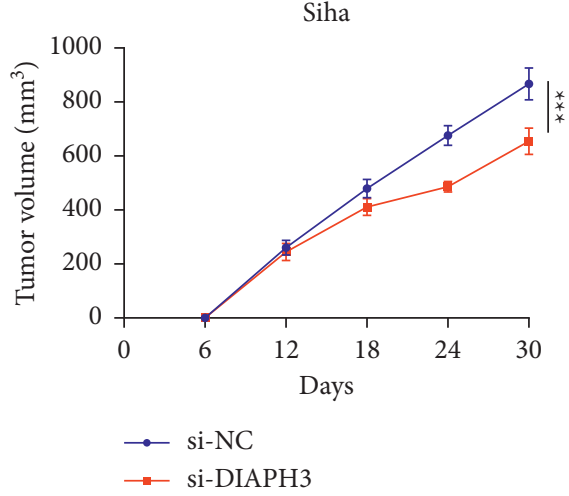

(f)

Figure 3: Knockdown of DIAPH3 suppressed the progression of CC in vivo. (a-c) The tumor volume and weight in the si-DiAPH3 group were obviously decreased in HeLa cells. $(\mathrm{d}-\mathrm{f})$ The tumor volume and weight in the si-DiAPH3 group were obviously decreased in Siha cells.

Meanwhile, we utilized the TIMER database to further analyze the correlation between the expression of DIAPH3 and diverse kinds of TIICs. Figure 7(a) shows that the expression of DIAPH3 was positively associated with the infiltrating levels of T cell CD8 + naive ( $\operatorname{cor}=0.13, p=3.04 \mathrm{e}-02$ ) and $\mathrm{T}$ cell CD4+ Th2 (cor $=0.309, p=1.48 \mathrm{e}-07)$ and negatively related to the infiltrating levels of B cells (cor $=-0.161$, $p=7.3 \mathrm{e}-03$ ), monocytes (cor $=-0.166, p=5.76 \mathrm{e}-03$ ), macrophages (cor $=-0.166, p=5.52 \mathrm{e}-03$ ), and myeloid dendritic cells (cor $=-0.198, p=9.48 \mathrm{e}-04$ ). We found that DIAPH3 expression is associated with part of the markers of different immune cells (Table 5), especially negatively related to the markers of B cell and M1 macrophages (Figure 8).
3.5. Correlation between DIAPH3 Expression and Immune Checkpoint. Firstly, we divided the expression of DIAPH3 in TCGA-CESC into high and low groups according to the median value. The results of the correlation analysis between immune cell subgroups and functions revealed that T_cell_costimulation, T_cell_coinhibition, checkpoint, APC_coinhibition, APC_costimulation, type I INF response, CCR, and parainflammation were significantly different between the lowand high-risk groups (Figure 9(a)). Simultaneously, in view of the increasing importance of immunotherapy based on checkpoint inhibitors in antitumor therapy, we probed the difference in the expression of immune checkpoints between the low-risk and high-risk groups. We found a significant 


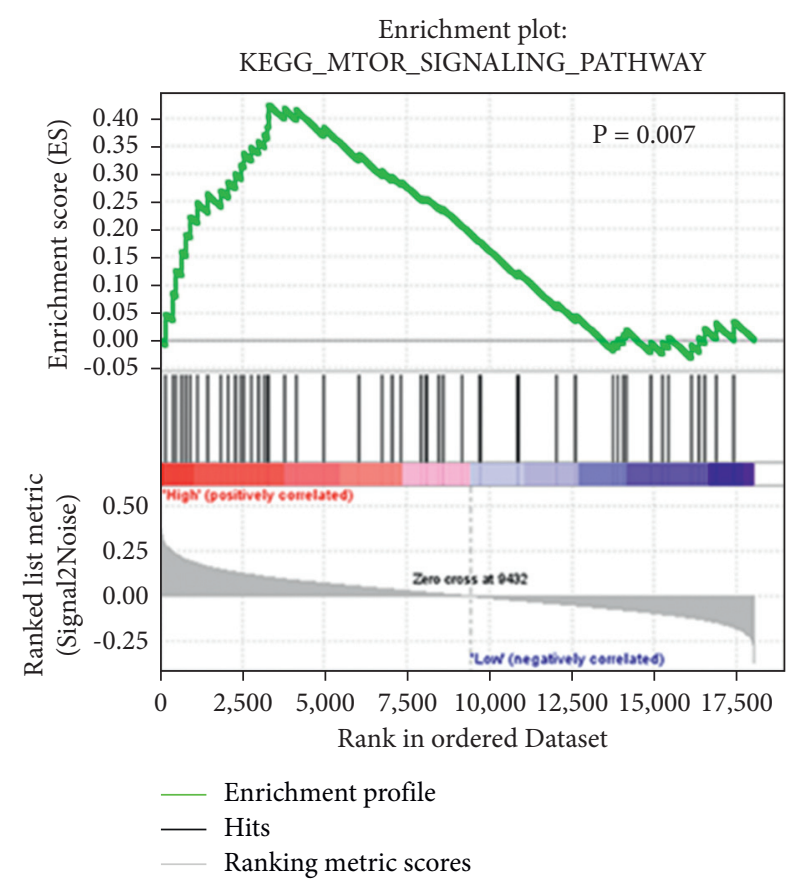

(a)
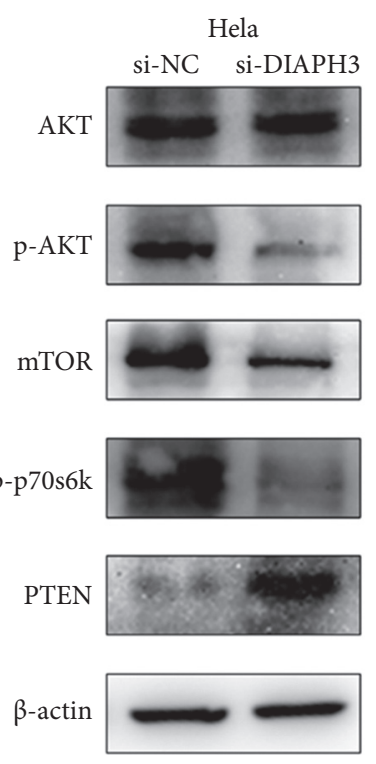

(c)

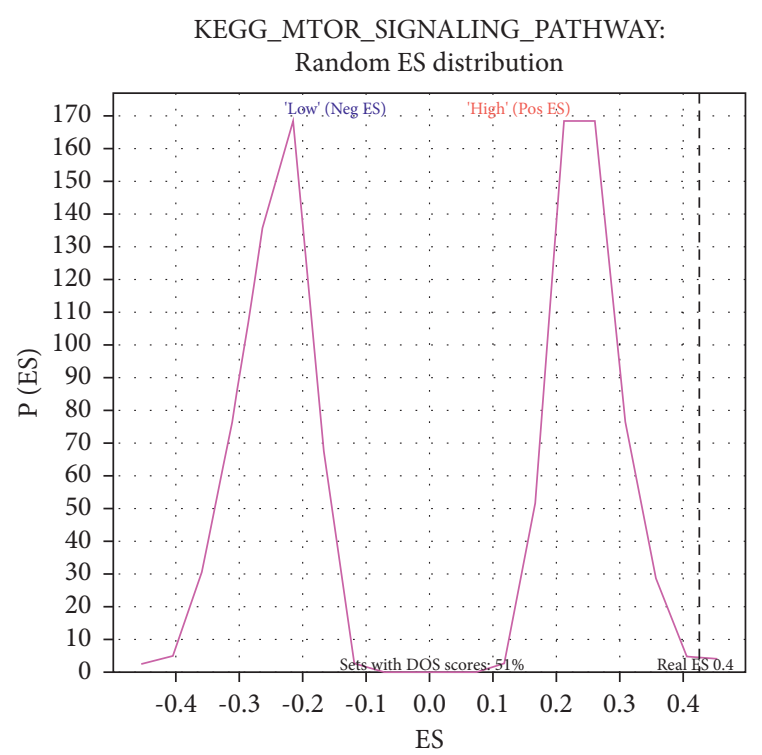

(b)

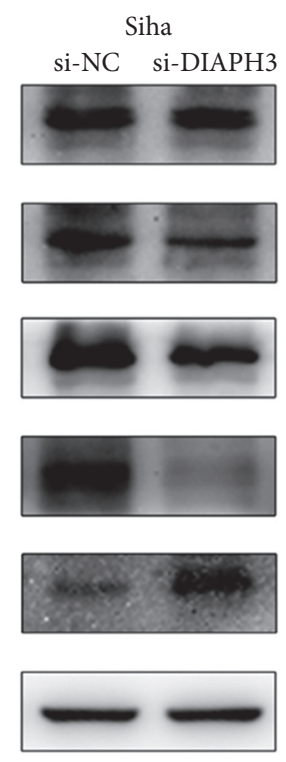

FIGURE 4: Knockdown of DIAPH3 inhibited cell proliferation through suppression of mTOR signaling pathway. (a, b) High expression of DIAPH3 was positively correlated with mTOR signaling pathway by GSEA. $P<0.01$. (c) Knockdown of DIAPH3 decreased the expression of related proteins p-AKT, mTOR, and p-p70s6k and increased the expression of PTEN in mTOR signaling pathway in HeLa and Siha cells.

difference in the expression of BTLA, CD48, PDCD-1, etc. between the low-risk and high-risk groups of patients (Figure 9(b)).

\section{Discussion}

Many researchers verified that DIAPH3 could regulate growth and migration by affecting cytoskeleton formation in different types of cancer $[11,12,18]$. And Hager $\mathrm{MH}$ et al. identified that DIAPH3 could accelerate the cellular transition to amoeboid tumor phenotype [19]. However, its role and molecular mechanism remain unknown in cervical cancer. We firstly reported that DIAPH3 was overexpressed in cervical cancer and enhanced the ability of CC cell proliferation. Moreover, we found that high expression of DIAPH3 indicated a poor prognosis.

The mTOR kinase is a core downstream molecule of PI3K/Akt to modulate cellular growth, metabolism, and migration, and its signaling pathway is frequently dysregulated in a variety of cancers [20-22]. Activation of the 


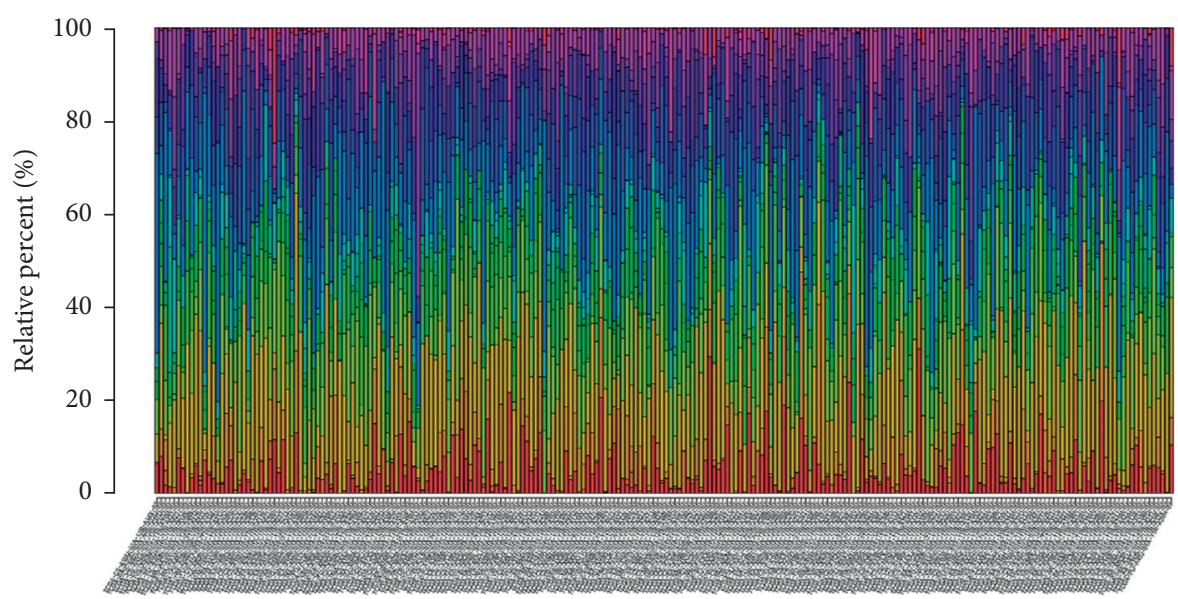

B cells naive
B cells memory
Plasma cells
T cells CD8
T cells CD4 naive
T cells CD4 memory resting
T cells CD4 memory activated
T cells follicular helper

Macrophages M2

Dendritic cells resting

- Dendritic cells activated

Mast cells resting

- Mast cells activated

Eosinophils

Neutrophils

(a)

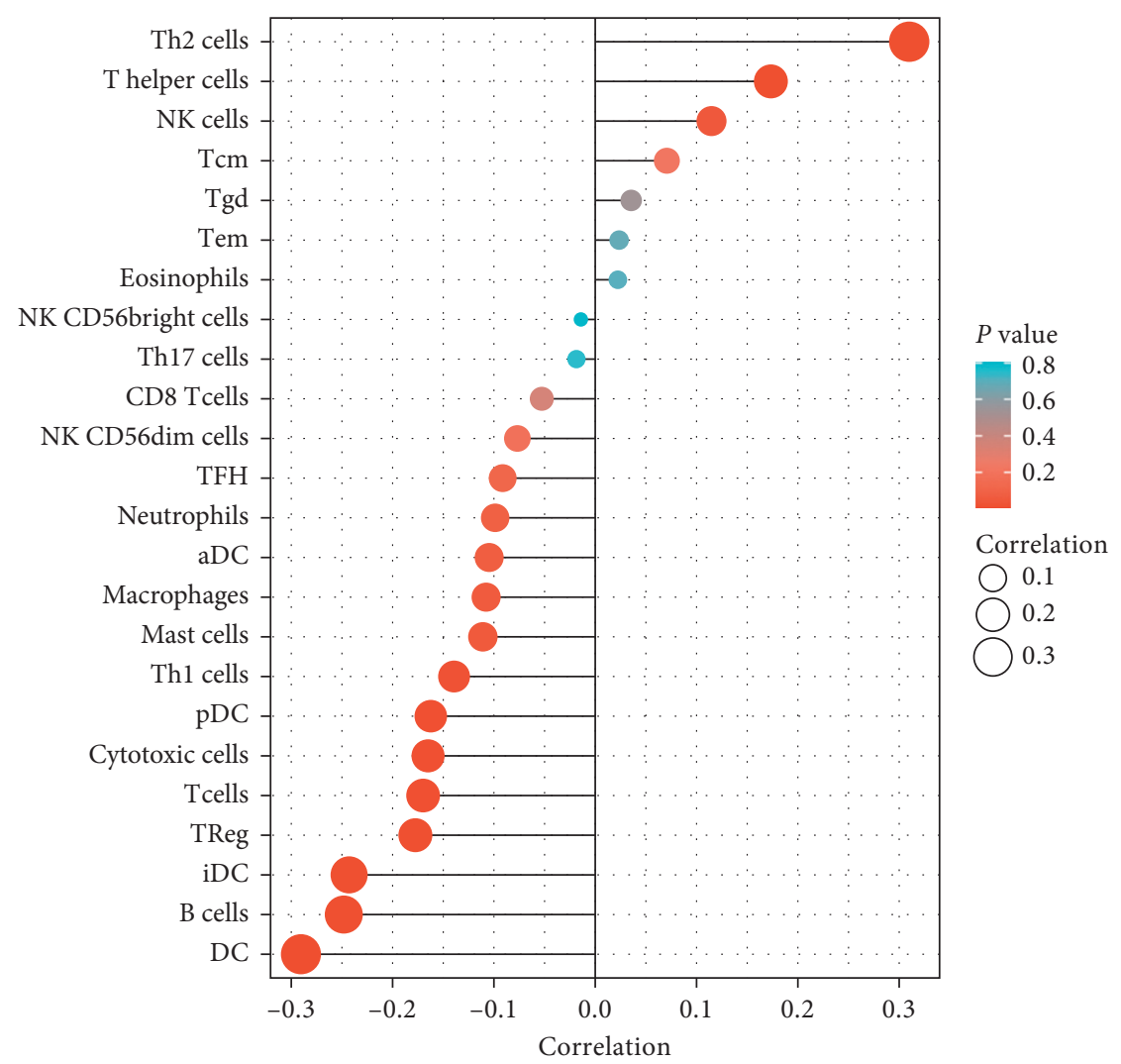

(b)

FIGURE 5: The fractions of tumor-infiltrating immune cells in CESC and correlation analysis with CIBERSORT. (a) The fractions of 22 kinds of tumor-infiltrating immune cells in CESC. (b) Association between the expression of DIAPH3 and relative abundance of 22 immune cells. The size of dots demonstrates the absolute value of Spearman $\mathrm{R}$. 
TABLE 4: Association between expression of DIAPH3 and immune infiltration.

\begin{tabular}{lccc}
\hline Gene & Cells & Correlation (Spearman) & $P$ value (Spearman) \\
\hline DIAPH3 & aDC & -0.104 & 0.068 \\
DIAPH3 & B cells & -0.248 & $<0.001$ \\
DIAPH3 & CD8 T cells & -0.053 & 0.357 \\
DIAPH3 & Cytotoxic cells & -0.165 & 0.004 \\
DIAPH3 & DC & -0.290 & $<0.001$ \\
DIAPH3 & Eosinophils & 0.022 & 0.699 \\
DIAPH3 & iDC & -0.243 & $<0.001$ \\
DIAPH3 & Macrophages & -0.108 & 0.060 \\
DIAPH3 & Mast cells & -0.111 & 0.053 \\
DIAPH3 & Neutrophils & -0.099 & 0.085 \\
DIAPH3 & NK CD56right cells & -0.014 & 0.807 \\
DIAPH3 & NK CD56dim cells & -0.077 & 0.179 \\
DIAPH3 & NK cells & 0.115 & 0.045 \\
DIAPH3 & pDC & -0.162 & 0.004 \\
DIAPH3 & T cells & -0.170 & 0.003 \\
DIAPH3 & Thelper cells & 0.173 & 0.002 \\
DIAPH3 & Tcm & 0.071 & 0.218 \\
DIAPH3 & Tem & 0.024 & 0.680 \\
DIAPH3 & TFH & -0.091 & 0.112 \\
DIAPH3 & Tgd & 0.036 & 0.536 \\
DIAPH3 & Th1 cells & -0.139 & 0.015 \\
DIAPH3 & Th17 cells & -0.019 & $<0.746$ \\
DIAPH3 & Th2 cells & 0.310 & $<001$ \\
DIAPH3 & TReg & -0.177 & 0.002 \\
\hline
\end{tabular}

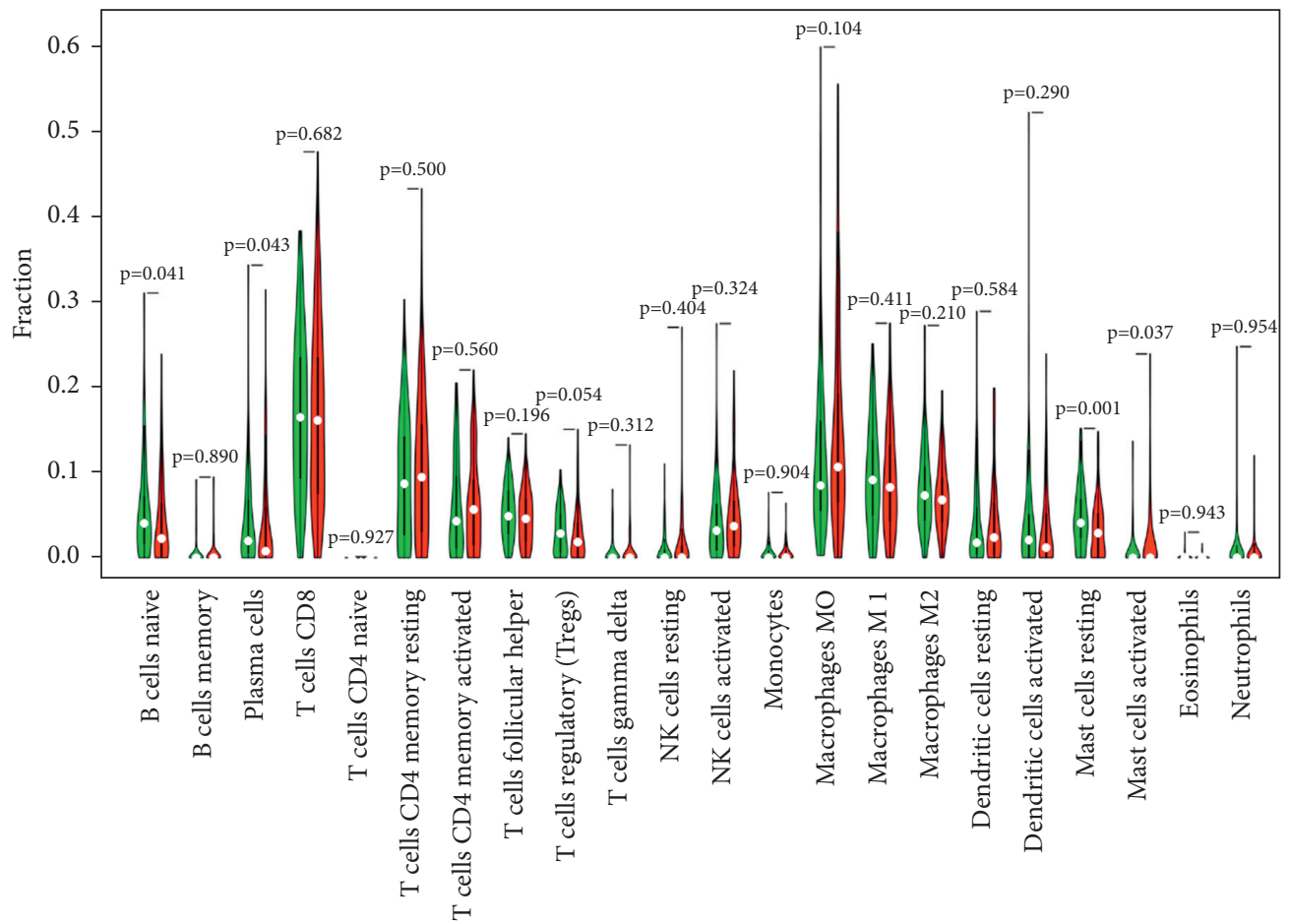

(a)

FIGURE 6: Continued. 

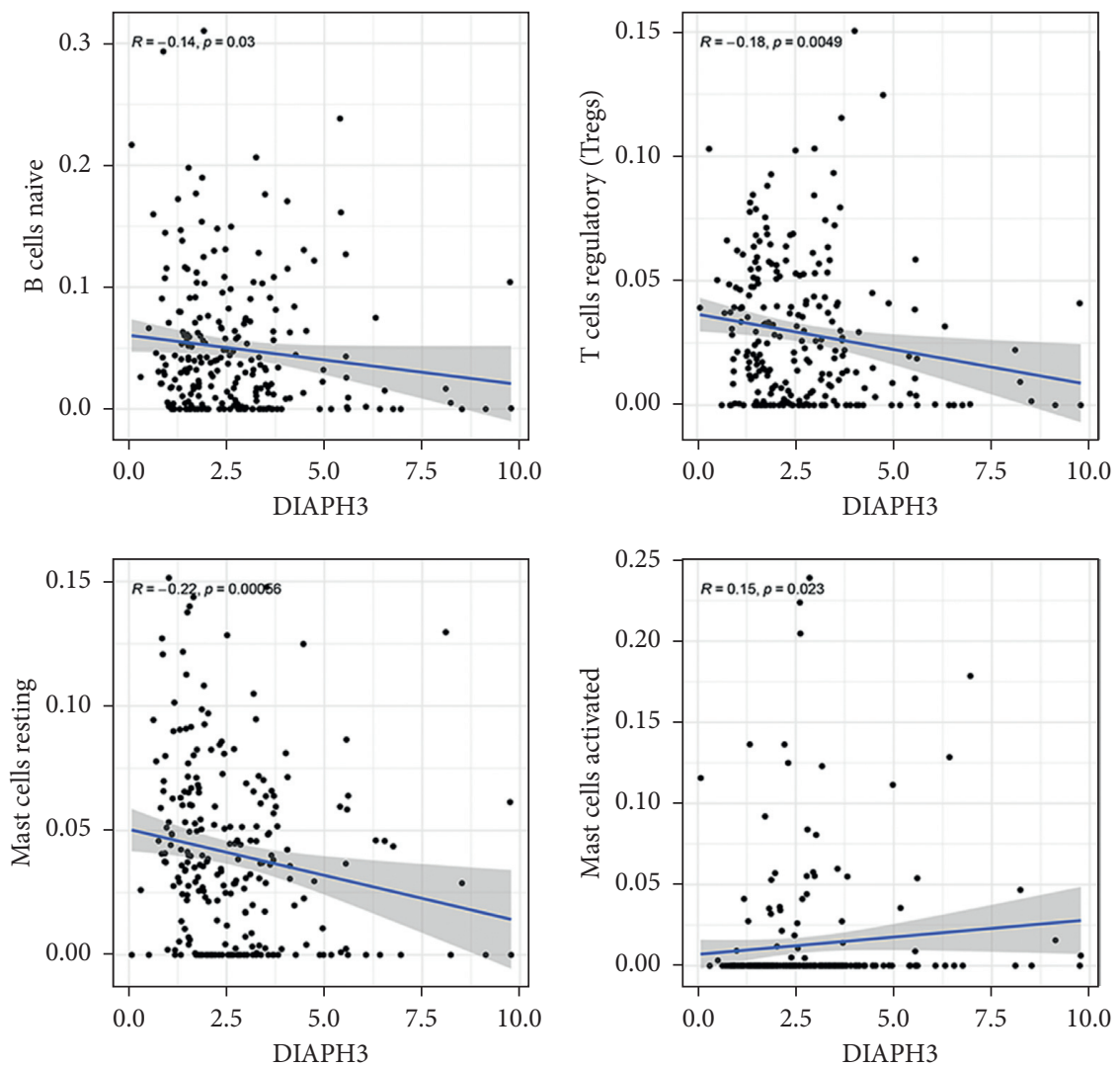

(b)

FIGURE 6: The correlation between the expression of DIAPH3 and fractions of tumor-infiltrating immune cells. (a) Comparison of 22 kinds of tumor-infiltrating immune cells between high and low DIAPH3 expression groups. The green violin diagram presents the low DIAPH3 expression group, and the red violin diagram presents the high DIAPH3 expression group. Wilcoxon rank-sum was used for the significance test. (b) The correlation between tumor-infiltrating immune cells and the expression of DIAPH3.
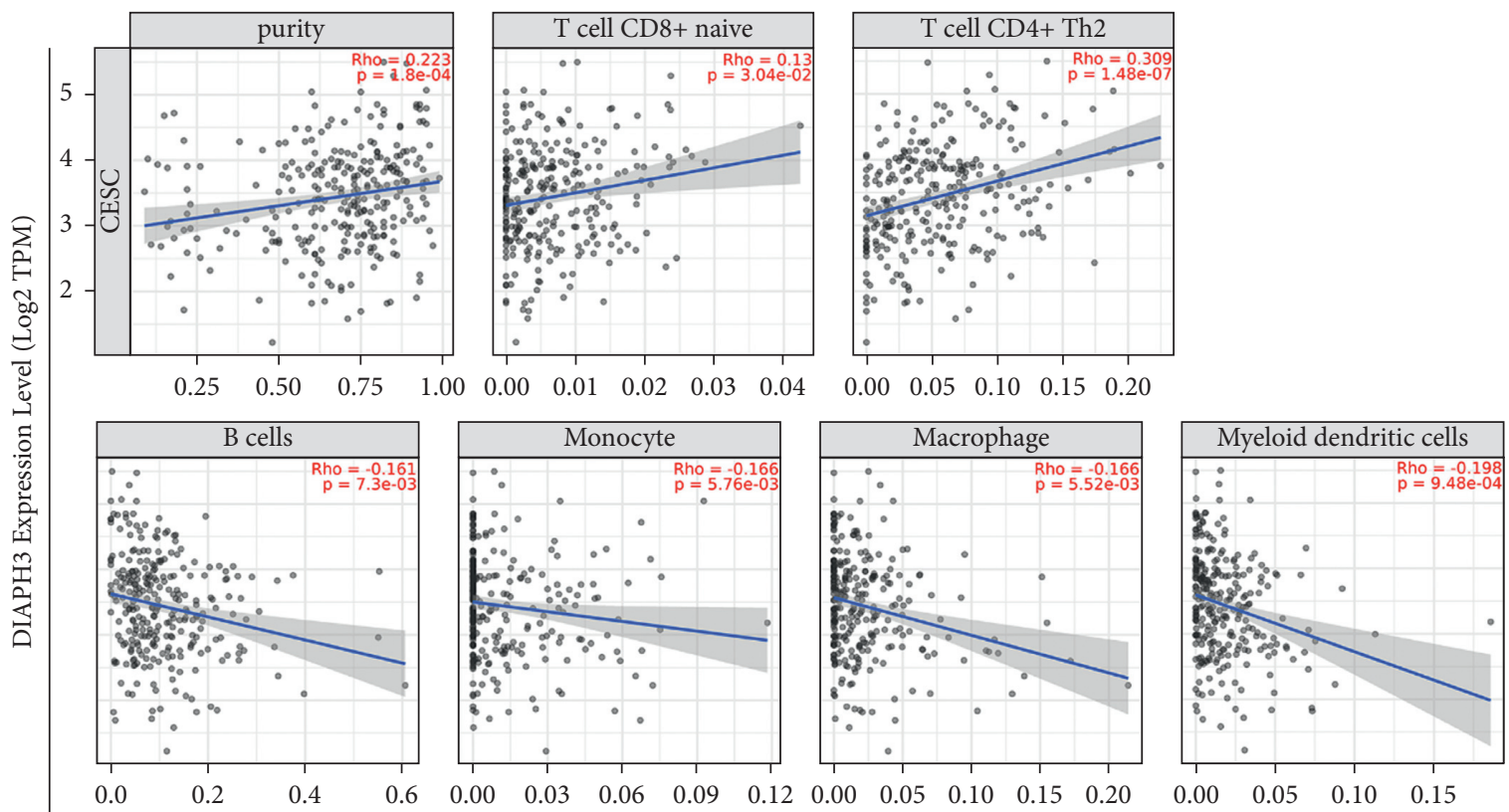

Infiltration level

FIGURE 7: Correlation of DIAPH3 expression with immune infiltration level in CESC with TIMER database. 
TABLE 5: Correlation analysis between DIAPH3 expression and related markers of immune cells using data in TIMER database.

\begin{tabular}{|c|c|c|c|}
\hline Description & Gene markers & $\begin{array}{c}\text { CESC } \\
\text { Cor }\end{array}$ & $\mathrm{P}$ \\
\hline \multirow{3}{*}{ CD8+ T cell } & CD8A & 0.013 & 0.835 \\
\hline & CD8B & 0.091 & 0.129 \\
\hline & CD45 & 0.027 & 0.649 \\
\hline \multirow{3}{*}{$\mathrm{T}$ cell (general) } & CD3D & 0.114 & 0.058 \\
\hline & CD3E & 0.098 & 0.104 \\
\hline & $\mathrm{CD} 2$ & 0.104 & 0.0827 \\
\hline \multirow{4}{*}{ B cell } & CD19 & 0.141 & $0.0193^{*}$ \\
\hline & CD79 A & -0.14 & $0.0198^{*}$ \\
\hline & CD27 & 0.112 & 0.0635 \\
\hline & $\mathrm{CD} 20$ & 0.113 & $0.0266^{*}$ \\
\hline \multirow{2}{*}{ Monocyte } & CD14 & 0.092 & 0.128 \\
\hline & CD115 (CSF1R) & 0.044 & 0.465 \\
\hline \multirow{3}{*}{ TAM } & CCL2 & 0.17 & $0.00463^{* *}$ \\
\hline & CD68 & 0.172 & $0.00411^{* *}$ \\
\hline & IL10 & 0.032 & 0.596 \\
\hline \multirow{5}{*}{ M1 macrophage } & INOS (NOS2) & 0.142 & $0.0184^{*}$ \\
\hline & CD80 & 0.022 & 0.717 \\
\hline & IRF5 & -0.14 & $0.0194^{*}$ \\
\hline & IL6 & 0.192 & $0.00134^{* *}$ \\
\hline & CD64 (FCGR1A) & 0.159 & $0.00789^{* *}$ \\
\hline \multirow{4}{*}{ M2 macrophage } & CD163 & -0.05 & 0.407 \\
\hline & CD206 & 0.011 & 0.853 \\
\hline & VSIG4 & 0.098 & 0.105 \\
\hline & MS4A4A & 0.055 & 0.362 \\
\hline \multirow{3}{*}{ Neutrophils } & CD66b (CEACAM8) & 0.013 & 0.825 \\
\hline & CD11b (ITGAM) & 0.056 & 0.357 \\
\hline & $\mathrm{CD} 15$ & 0.236 & $0.0000707^{* * *}$ \\
\hline \multirow{6}{*}{ Natural killer cell } & KIR2DL1 & 0.05 & 0.406 \\
\hline & KIR2DL3 & 0.025 & 0.678 \\
\hline & KIR3DL1 & 0.004 & 0.953 \\
\hline & KIR3DL2 & 0.01 & 0.873 \\
\hline & CD56 & 0.009 & 0.884 \\
\hline & CD335 (NKp46) & 0.004 & 0.942 \\
\hline \multirow{5}{*}{ Dendritic cell } & BDCA-1 (CD1C) & 0.061 & 0.316 \\
\hline & BDCA-3 (CD141) & -0.05 & 0.41 \\
\hline & BDCA-4 (NRP1) & 0.036 & 0.549 \\
\hline & CD123 & 0.045 & 0.453 \\
\hline & CD11c (ITGAX) & 0.083 & 0.168 \\
\hline \multirow{3}{*}{ Th1 } & T-bet (TBX21) & 0.071 & 0.237 \\
\hline & STAT4 & -0.005 & 0.931 \\
\hline & STAT1 & 0.017 & 0.78 \\
\hline \multirow{3}{*}{ Th2 } & GATA3 & 0.068 & 0.26 \\
\hline & STAT6 & 0.011 & 0.857 \\
\hline & IL13 & 0.025 & 0.68 \\
\hline \multirow{2}{*}{ Tfh } & BCL6 & 0.064 & 0.285 \\
\hline & IL21 & 0.066 & 0.275 \\
\hline \multirow{2}{*}{ Th17 } & STAT3 & 0.028 & 0.645 \\
\hline & IL17A & 0.018 & 0.767 \\
\hline \multirow{4}{*}{ Treg } & FOXP3 & 0.019 & 0.75 \\
\hline & CD25 & 0.014 & 0.813 \\
\hline & CCR8 & 0.014 & 0.821 \\
\hline & STAT5B & 0.066 & 0.274 \\
\hline \multirow{4}{*}{ T cell exhaustion } & PD-1 (PDCD1) & 0.064 & 0.289 \\
\hline & CTLA4 & 0.035 & 0.567 \\
\hline & LAG3 & 0.036 & 0.548 \\
\hline & TIM-3 (HAVCR2) & 0.092 & 0.128 \\
\hline
\end{tabular}

${ }^{*} p<0.05 ;{ }^{*}{ }^{*} p<0.01 ;{ }^{*}{ }^{*} p<0.001$. 


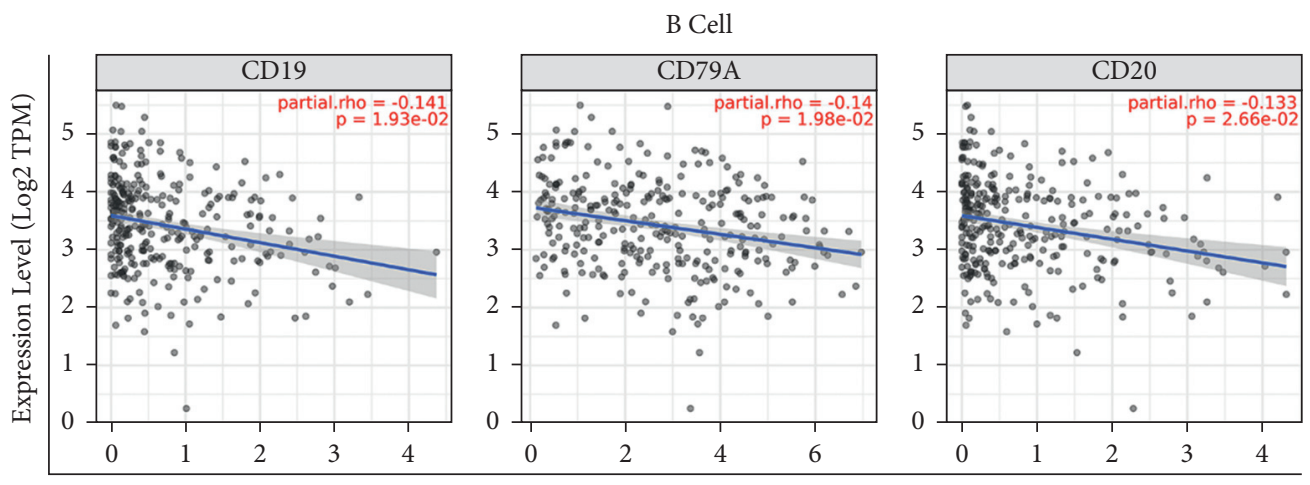

Expression Level (Log2 TPM)
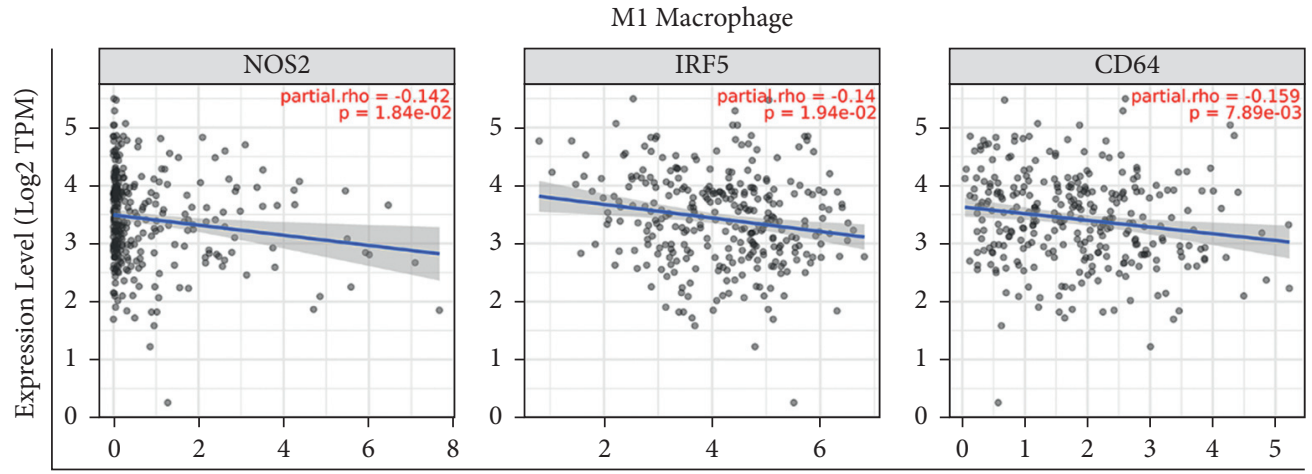

Expression Level (Log2 TPM)

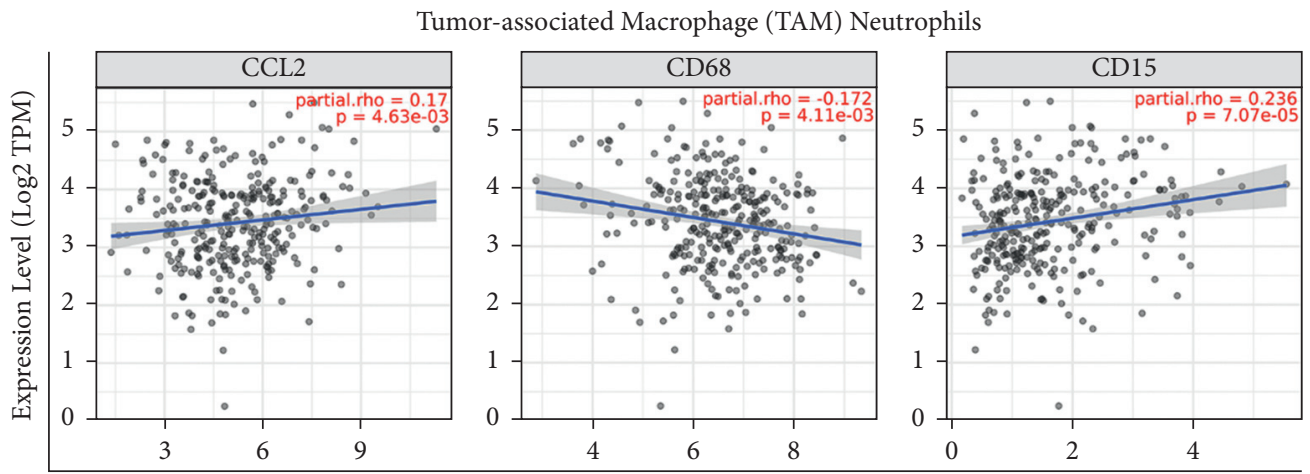

Expression Level (Log2 TPM)

Figure 8: Correlation of DIAPH3 expression with marker genes of B cells, M1 macrophages, tumor-associated macrophage (TAM), and neutrophils in CESC with TIMER database.

mTOR signaling pathway has been observed in cervical cancer $[23,24]$. The inhibition of mTOR by rapamycin and its analogs seems to be effective in cancer treatment [25]. Importantly, therapy targeting the mTOR signaling pathway indicates clinical benefits in cervical cancer [26]. In our study, we firstly explored the notion that knockdown of DIAPH3 inhibits the proliferation of cervical cancer cells through inactivating the mTOR signaling pathway.

Recently, studies have reported that TIICs could regulate tumor progression [27]. Additionally, the poor prognosis of tumor patients is associated with the accumulation of TIICs in HCC [28]. In the current study, we revealed that the expression of DIAPH3 in CC was negatively associated with some innate immunocytes infiltration, such as B cells, DCs, and cytotoxic cells. Three new studies reported that, with the presence of the two key elements (B cells and tertiary lymphatic structure) in tumors, cancer patients will have better results when they receive immunotherapy [28-31]. DCs can significantly affect the regulation of cancer immune response [32]. Moreover, cytotoxic cells are also critical antitumor cells. A study reported that HCC cells suppressed cytotoxic T cells to modulate tumor resistance to PD1 [33]. In summary, the above results indicated that DIAPH3 plays important roles in the regulation of TIICs in CC. We hope 


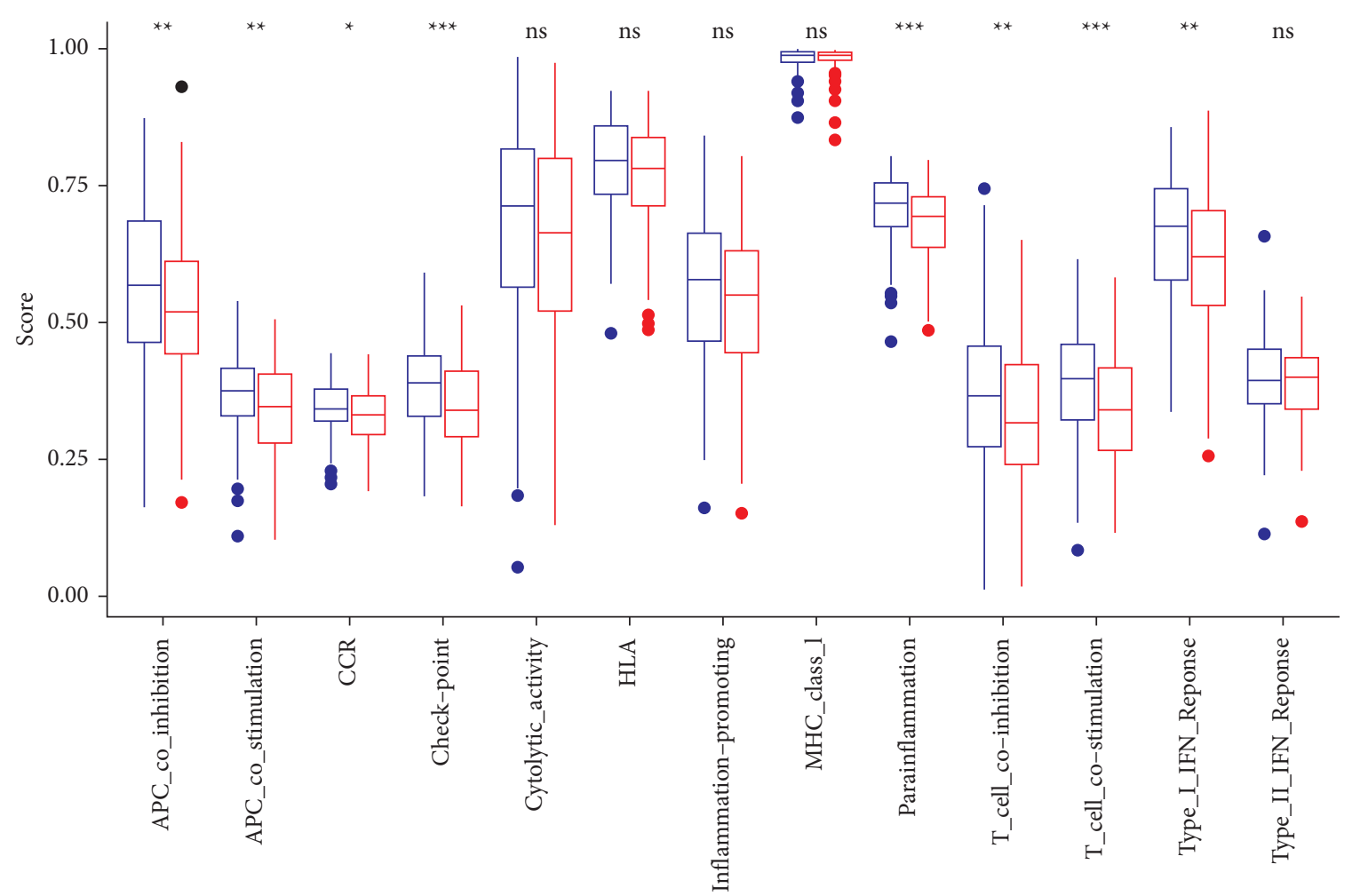

DIAPH3 expression

追 low

官 high

(a)

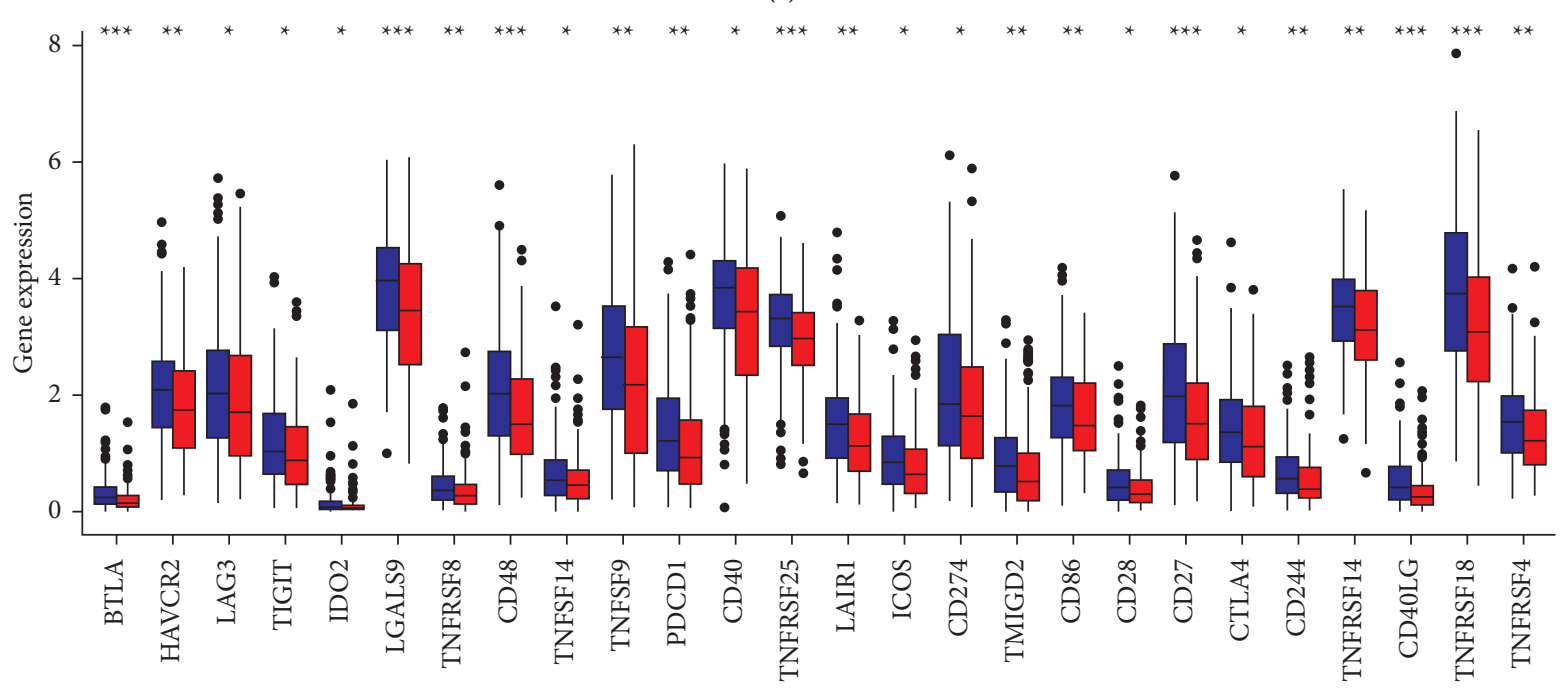

Risk

투 low

官 high

(b)

FIGURE 9: Correlation between the expression of DIAPH3 and the immune checkpoint. (a) ssGSEA for the association between immune cell subpopulations and related functions. (b) Expression of immune checkpoints among high- and low-risk DIAPH3 groups. 
that DIAPH3 may become a novel therapy target in cervical cancer.

\section{Data Availability}

The data used to support the findings of this study are available from the corresponding author upon request.

\section{Conflicts of Interest}

The authors declare that they have no conflicts of interest.

\section{Authors' Contributions}

L. Wan and J. Zhu are contributed equally to this work.

\section{Acknowledgments}

The authors thank LetPub (http://www.letpub.com) for paper linguistic assistance.

\section{References}

[1] R. L. Siegel, K. D. Miller, and A. Jemal, "Cancer statistics, 2019,” CA: A Cancer Journal for Clinicians, vol. 69, no. 1, pp. 7-34, 2019.

[2] W. Chen, R. Zheng, P. D. Baade et al., "Cancer statistics in China, 2015," CA: A Cancer Journal for Clinicians, vol. 66, no. 2, pp. 115-132, 2016.

[3] E. J. Crosbie, M. H. Einstein, S. Franceschi, and H. C. Kitchener, "Human papillomavirus and cervical cancer," The Lancet, vol. 382, no. 9895, pp. 889-899, 2013.

[4] W. Small Jr., M. A. Bacon, A. Bajaj et al., "Cervical cancer: a global health crisis," Cancer, vol. 123, no. 13, pp. 2404-2412, 2017.

[5] P. Petignat and M. Roy, "Diagnosis and management of cervical cancer," BMJ, vol. 335, no. 7623, pp. 765-768, 2007.

[6] M. A. Chesarone, A. G. DuPage, and B. L. Goode, "Unleashing formins to remodel the actin and microtubule cytoskeletons," Nature Reviews Molecular Cell Biology, vol. 11, no. 1, pp. 62-74, 2010.

[7] M. Katoh and M. Katoh, "Identification and characterization of human DIAPH3 gene in silico," International Journal of Molecular Medicine, vol. 13, pp. 473-478, 2004.

[8] B. J. Wallar and A. S. Alberts, "The formins: active scaffolds that remodel the cytoskeleton," Trends in Cell Biology, vol. 13, no. 8, pp. 435-446, 2003.

[9] C. H. Eng, T. M. Huckaba, and G. G. Gundersen, “The formin mDia regulates GSK $3 \beta$ through novel PKCs to promote microtubule stabilization but not MTOC reorientation in migrating fibroblasts," Molecular Biology of the Cell, vol. 17, no. 12, pp. 5004-5016, 2006.

[10] D. Di Vizio, J. Kim, M. H. Hager et al., "Oncosome formation in prostate cancer: association with a region of frequent chromosomal deletion in metastatic disease," Cancer Research, vol. 69, no. 13, pp. 5601-5609, 2009.

[11] J. Jiang, "Diaphanous-related formin-3 overexpression inhibits the migration and invasion of triple-negative breast cancer by inhibiting RhoA-GTP expression," Biomedicine \& Pharmacotherapy, vol. 94, pp. 439-445, 2017.

[12] L. Dong, Z. Li, L. Xue et al., "DIAPH3 promoted the growth, migration and metastasis of hepatocellular carcinoma cells by activating beta-catenin/TCF signaling," Molecular and Cellular Biochemistry, vol. 438, no. 1-2, pp. 183-190, 2018.

[13] S.-R. Woo, L. Corrales, and T. F. Gajewski, "Innate immune recognition of cancer," Annual Review of Immunology, vol. 33, no. 1, pp. 445-474, 2015.

[14] L. Corrales, K. Scilla, C. Caglevic, K. Miller, J. Oliveira, and C. Rolfo, "Immunotherapy in lung cancer: a new age in cancer treatment," Advances in Experimental Medicine \& Biology, vol. 995, pp. 65-95, 2018.

[15] P. Isaacsson Velho and E. S. Antonarakis, "PD-1/PD-L1 pathway inhibitors in advanced prostate cancer," Expert Review of Clinical Pharmacology, vol. 11, no. 5, pp. 475-486, 2018.

[16] D. Liu, B. Schilling, D. Liu et al., "Integrative molecular and clinical modeling of clinical outcomes to PD1 blockade in patients with metastatic melanoma," Nature Medicine, vol. 25, no. 12, pp. 1916-1927, 2019.

[17] S. L. Topalian, F. S. Hodi, J. R. Brahmer et al., "Safety, activity, and immune correlates of anti-PD-1 antibody in cancer," New England Journal of Medicine, vol. 366, no. 26, pp. 2443-2454, 2012.

[18] A. Schönichen and M. Geyer, "Fifteen formins for an actin filament: a molecular view on the regulation of human formins," Biochimica et Biophysica Acta (BBA) - Molecular Cell Research, vol. 1803, no. 2, pp. 152-163, 2010.

[19] M. H. Hager, S. Morley, D. R. Bielenberg et al., "DIAPH3 governs the cellular transition to the amoeboid tumour phenotype," EMBO Molecular Medicine, vol. 4, no. 8, pp. 743-760, 2012.

[20] D. A. Guertin and D. M. Sabatini, "Defining the role of mTOR in cancer," Cancer Cell, vol. 12, no. 1, pp. 9-22, 2007.

[21] R. Iglesias-Bartolome, V. Patel, A. Cotrim et al., "mTOR inhibition prevents epithelial stem cell senescence and protects from radiation-induced mucositis," Cell Stem Cell, vol. 11, no. 3, pp. 401-414, 2012.

[22] I. Vivanco and C. L. Sawyers, "The phosphatidylinositol 3Kinase-AKT pathway in human cancer," Nature Reviews Cancer, vol. 2, no. 7, pp. 489-501, 2002.

[23] J. Ji and P.-S. Zheng, "Activation of mTOR signaling pathway contributes to survival of cervical cancer cells," Gynecologic Oncology, vol. 117, no. 1, pp. 103-108, 2010.

[24] M.-K. Kim, T.-J. Kim, C. O. Sung et al., "High expression of $\mathrm{mTOR}$ is associated with radiation resistance in cervical cancer," Journal of Gynecologic Oncology, vol. 21, no. 3, pp. 181-185, 2010.

[25] A. Panwalkar, S. Verstovsek, and F. J. Giles, "Mammalian target of rapamycin inhibition as therapy for hematologic malignancies," Cancer, vol. 100, no. 4, pp. 657-666, 2004.

[26] M.-M. Hou, X. Liu, J. Wheler et al., "Targeted PI3K/AKT/ mTOR therapy for metastatic carcinomas of the cervix: A phase I clinical experience," Oncotarget, vol. 5, no. 22, pp. 11168-11179, 2014.

[27] P. Domingues, M. González-Tablas, Á. Otero et al. "Tumor infiltrating immune cells in gliomas and meningiomas," Brain, Behavior, and Immunity, vol. 53, pp. 1-15, 2016.

[28] H. Sun, L. Liu, Q. Huang et al., "Accumulation of tumorinfiltrating CD49a+ NK cells correlates with poor prognosis for human hepatocellular carcinoma," Cancer Immunology Research, vol. 7, no. 9, pp. 1535-1546, 2019.

[29] R. Cabrita, M. Lauss, A. Sanna et al., "Tertiary lymphoid structures improve immunotherapy and survival in melanoma," Nature, vol. 577, no. 7791, pp. 561-565, 2020. 
[30] B. A. Helmink, S. M. Reddy, J. Gao et al., "B cells and tertiary lymphoid structures promote immunotherapy response," Nature, vol. 577, no. 7791, pp. 549-555, 2020.

[31] F. Petitprez, A. de Reyniès, E. Z. Keung et al., "B cells are associated with survival and immunotherapy response in sarcoma," Nature, vol. 577, no. 7791, pp. 556-560, 2020.

[32] S. K. Wculek, F. J. Cueto, A. M. Mujal, I. Melero, M. F. Krummel, and D. Sancho, "Dendritic cells in cancer immunology and immunotherapy," Nature Reviews Immunology, vol. 20, no. 1, pp. 7-24, 2020.

[33] D. K.-C. Chiu, V. W.-H. Yuen, J. W.-S. Cheu et al., "Hepatocellular carcinoma cells up-regulate PVRL1, stabilizing PVR and inhibiting the cytotoxic T-cell response via TIGIT to mediate tumor resistance to PD1 inhibitors in mice," Gastroenterology, vol. 159, no. 2, pp. 609-623, 2020. 